\title{
Relação da Função Pulmonar e da Força Inspiratória com Capacidade Aeróbica e com Prognóstico na Insuficiência Cardíaca
}

\author{
Relationship of Lung Function and Inspiratory Strength with Exercise Capacity and Prognosis in Heart Failure
}

\author{
Sergio Henrique Rodolpho Ramalho, ${ }^{\circledR}$ Alexandra Correa Gervazoni Balbuena de Lima, ${ }^{1}$ Fabiola Maria Ferreira \\ da Silva, ${ }^{1}$ Fausto Stauffer Junqueira de Souza, ${ }^{1}$ Lawrence Patrick Cahalin, ${ }^{3}$ Graziella França Bernardelli Cipriano, ${ }^{1,2}$ \\ Gerson Cipriano Junior ${ }^{1,2} \bullet$ \\ Programa de Pós-graduação em Ciências e Tecnologias em Saúde - Universidade de Brasília, Brasília, ${ }^{1}$ DF - Brasil \\ Programa de Pós-graduação em Ciências da Reabilitação - Universidade de Brasília, ${ }^{2}$ Brasília, DF - Brasil \\ Universidade de Miami - Miller School of Medicine, ${ }^{3}$ Miami - EUA
}

\section{Resumo}

Fundamento: A espirometria é subutilizada na insuficiência cardíaca (IC) e não está claro o grau de associação de cada defeito com a capacidade de exercício e com o prognóstico desses pacientes.

Objetivo: Determinar a relação da \%CVF prevista (ppCVF) e do $\mathrm{VEF}_{1} / \mathrm{CVF}$ contínuos com: 1) pressão inspiratória máxima (PImáx), fração de ejeção do ventrículo esquerdo (FEVE) e desempenho ao exercício; e 2) prognóstico, para o desfecho composto de morte cardiovascular, transplante cardíaco ou implante de dispositivo de assistência ventricular.

Métodos: Coorte de 111 participantes com IC (estágios AHA C/D) sem pneumopatia; foram submetidos a espirometria, manovacuometria e teste cardiopulmonar máximo. As magnitudes de associação foram verificadas por regressões lineares e de Cox (HR; IC 95\%), ajustadas para idade/sexo, e p <0,05 foi considerado significativo.

Resultados: Com idade média $57 \pm 12$ anos, $60 \%$ eram homens, $64 \%$ em NYHAIII. A cada aumento de $10 \%$ no VEF $/ C V F$ [ $\beta$ 7\% (IC 95\%: 3-10)] e no ppCVF [4\% (2-6)], foi associado à reserva ventilatória (VRes); no entanto, apenas o ppCVF associado à PImáx $\left[3,8 \mathrm{cmH}_{2} \mathrm{O}(0,3-7,3)\right]$, à fração de ejeção do ventrículo esquerdo (FEVE) $[2,1 \%(0,5-3,8)]$ e ao VO pico $[0,5 \mathrm{~mL} / \mathrm{kg} / \mathrm{min}(0,1-1,0)]$, considerando idade/sexo. Em 2,2 anos (média), ocorreram 22 eventos; tanto FEV1/FVC (HR 1,44; IC 95\%: 0,97-2,13) quanto ppCVF (HR 1,13; 0,89-1,43) não foram associados ao desfecho. Apenas no subgrupo FEVE $\leq 50 \%\left(n=87,20\right.$ eventos), $\operatorname{VEF}_{1} / C V F(H R$ 1,50; 1,01-2,23), mas não ppCVF, foi associado a risco.

Conclusão: Na IC crônica, ppCVF reduzido associou-se a menor PImáx, FEVE, VRes e $\mathrm{VO}_{2}$ pico, mas não distinguiu pior prognóstico em 2,2 anos de acompanhamento. Entretanto, VEF $/$ CVF associou-se apenas com VRes, e, em participantes com $\mathrm{FEVE} \leq \mathbf{5 0} \%$, o $\mathrm{VEF}_{1} / \mathrm{CVF}$ reduzido mostrou pior prognóstico proporcional. Portanto, VEF $/ \mathrm{CVF}^{\mathrm{e}}$ ppFVC contribuem para melhor fenotipagem de pacientes com IC.

Palavras-chave: Insuficiência Respiratória; Músculos Respiratórios; Função Ventricular; Tolerância ao Exercício; Medição de Risco.

\footnotetext{
Abstract

Background: Spirometry is underused in heart failure (HF) and the extent to which each defect associates with exercise capacity and prognosis is unclear. Objective: To determine the distinct relationship of continuous \%predicted FVC (ppFVC) and FEV /FVC with: 1) maximal inspiratory pressure (MIP), left ventricular ejection fraction (LVEF), exercise performance; and 2) prognosis for the composite of cardiovascular death, heart transplantation or left ventricular assist device implant.
}

Methods: A cohort of 111 HF participants (AHA stages C/D) without diagnosed pneumopathy, spirometry, manovacuometry and maximum cardiopulmonary test. The association magnitudes were verified by linear and Cox (HR; 95\% CI) regressions, age/sex adjusted. A p $<0.05$ was considered significant.

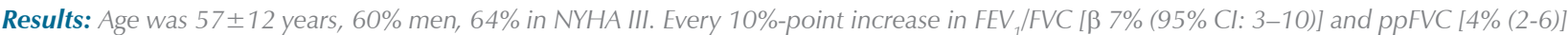
associated with ventilatory reserve (VRes), however only ppFVC associated with MIP [3.8 $\left.\mathrm{CmH}_{2} \mathrm{O}(0.3-7.3)\right]$, LVEF [2.1\% (0.5-3.8)] and VO $\mathrm{p}_{2}$ eak $[0.5 \mathrm{~mL} / \mathrm{kg} / \mathrm{min}(0.1-1.0)]$, accounting for age/sex. In 2.2 years (mean), 22 events occurred, and neither FEV $/$ /FVC (HR 1.44; 95\% Cl: 0.97-2.13) nor ppFVC (HR 1.13; 0.89-1.43) was significantly associated with the outcome. Only in the LVEF $\leq 50 \%$ subgroup ( $n=87,20$ events), FEV /FVC (HR 1.50; 1.01-2.23), but not ppFVC, was associated with greater risk.

Correspondência: Sergio Henrique Rodolpho Ramalho, MD, MSc •

Universidade de Brasília - Faculdade de Ceilândia - Laboratório de Fisiologia - Campus Universitário, Centro Metropolitano - CEP $72220-275$,

Ceilândia Sul, Brasílie, DF - Brasil

E-mail: shrramalho@gmail.com

Artigo recebido em 20/10/2020, revisado em 02/04/2021, aceito em 12/05/2021

DOI: https://doi.org/10.36660/abc.20201130 
Conclusions: In chronic HF, reduced ppFVC associated with lower MIP, LVEF, VRes and VO peak, but no distinct poorer prognosis over 2.2 years of follow-up. Distinctively, FEV $/$ /FVC was associated only with VRes, and, in participants with LVEF $\leq 50 \%$, FEV $V_{1} / F V C$ reduction proportionally worsened prognosis. Therefore, FEV / /FVC and ppFVC add supplementary information regarding HF phenotyping.

Keywords: Respiratory Insufficiency; Respiratory Muscles; Ventricular Function; Exercise Tolerance; Risk Assessment.

Full texts in English - http://www.arquivosonline.com.br

\section{Introdução}

A insuficiência cardíaca (IC) e a disfunção pulmonar frequentemente coexistem, emergindo de vários mecanismos: espessamento septal e congestão parenquimatosa; redução da função vascular pulmonar e hipoperfusão microvascular; desregulação e remodelação das vias aéreas; fraqueza muscular inspiratória e periférica; desequilíbrio dos quimio, ergo e metaborreflexos para controle ventilatório; cardiomegalia; e diminuição da condutância brônquica. ${ }^{1-3}$ No entanto, a espirometria é amplamente subutilizada na IC. Mesmo quando IC e doença pulmonar obstrutiva crônica (DPOC) coexistem, 80\% dos indivíduos realizam ecocardiografia, mas $<50 \%$ realizam espirometria. ${ }^{4-6}$

Alterações ventilatórias subclínicas estão presentes nos estágios iniciais da IC, contribuindo para dispneia e intolerância ao exercício. ${ }^{3}$ A obstrução das vias aéreas pode ser encontrada principalmente em pacientes não compensados, e defeitos restritivos são descritos, sobretudo, em indivíduos crônicos e estáveis. ${ }^{7,8}$ O reconhecimento de alterações espirométricas de base fundamenta a interpretação do teste de exercício cardiopulmonar (CPX) para o diagnóstico diferencial de limitação de esforço, ${ }^{8,9}$ e também identifica o risco de mortalidade em IC com fração de ejeção preservada (ICFEP) ou IC com fração de ejeção reduzida (ICFER). ${ }^{10,11}$

No entanto, a associação dos parâmetros espirométricos com a limitação do exercício e prognóstico na IC ainda é controversa, ${ }^{8}$ dado o seu uso em diferentes fenótipos e estados de gravidade da IC, a possível contribuição diferencial de cada defeito obstrutivo e restritivo e as potenciais relações não lineares e pouco exploradas entre as disfunções pulmonares e cardíacas. ${ }^{12}$ Nossa hipótese é que, na IC estável crônica, o comprometimento da capacidade vital forçada (CVF) e da relação do volume expirado forçado em 1 segundo $\left(\mathrm{VEF}_{1}\right) /$ CVF se associam de forma diferente com outros parâmetros funcionais em repouso e no exercício e, consequentemente, com pior prognóstico. Portanto, os objetivos foram (1) definir até que ponto o $\mathrm{VEF}_{1} / \mathrm{CVF}$ e CVF se associam com a fração de ejeção do ventrículo esquerdo (FEVE), força respiratória e respostas ao exercício; e (2) determinar suas associações com a incidência de eventos cardiovasculares maiores (morte cardiovascular, transplante cardíaco e dispositivo de assistência ventricular esquerda (DAVE).

\section{Métodos}

\section{População do estudo e características clínicas}

Esta coorte incluiu 158 pacientes consecutivos com IC encaminhados ao laboratório de fisiologia (Universidade de Brasília, Brasília, Brasil) para CPX de junho de 2015 a julho de 2016, que foram seguidos até julho de
2018, correspondendo a pelo menos 24 meses de acompanhamento. Pacientes diagnosticados com IC, independentemente da etiologia ou FEVE, deveriam estar clinicamente estáveis por pelo menos 3 meses antes da inclusão no estudo (sem descompensação ou hospitalização), sem doença pulmonar diagnosticada (asma, DPOC, enfisema ou em uso de broncodilatadores), sem condições clinicas que impossibilitassem um CPX máximo em cicloergômetro. Os participantes foram submetidos à ecocardiograma (HD 11XE, Phillips, Amsterdam, Holanda) até 1 mês antes da inclusão. Para esta análise, incluímos 111 pacientes com IC, considerando dados espirométricos incompletos em 43 participantes, e 4 não conseguiram manobras com qualidade mínima para interpretação adequada. ${ }^{13}$

No primeiro dia, os indivíduos foram submetidos à avaliação clínica, seguida de avaliação da força respiratória e espirometria após 30 minutos de repouso. O CPX foi realizado no dia seguinte. O ecocardiograma foi realizado de acordo com as recomendações; ${ }^{14}$ a pressão sistólica da artéria pulmonar (PSAP) foi estimada a partir da velocidade de pico do jato de regurgitação tricúspide com Doppler, quando disponível. Hipertensão e diabetes foram definidos com base no autorrelato, uso de medicamentos ou medidas elevadas na consulta (pressão arterial $\geq 140 / 90 \mathrm{mmHg}$ e glicemia de jejum $\geq 126$ ou glicose aleatória $\geq 200 \mathrm{mg} / \mathrm{dL}$, respectivamente). Dislipidemia foi definida como LD L $\geq 160 \mathrm{mg} / \mathrm{dL}$ ou uso de hipolipemiante. O tabagismo foi autorrelatado. O cardiologista assistente informou a etiologia primária da IC e a prescrição farmacológica.

Todos os participantes assinaram um termo de consentimento informado e a aprovação do conselho de revisão institucional foi obtida no Comitê de Ética em Pesquisa da Universidade de Brasília (CAAE 50414115.4.0000.0030).

\section{Avaliação da função pulmonar e força respiratória}

A espirometria foi realizada de acordo com as recomendações-padrão. ${ }^{13} \mathrm{VEF}_{1}$ foi obtido a partir do volume de gás exalado no primeiro segundo da expiração. A CVF foi obtida a partir do volume de gás vigorosamente exalado após uma inspiração máxima (Microlab, Carefusion, Yorba Linda, USA). A melhor de cinco tentativas foi utilizada. Os parâmetros de referência derivaram de equações brasileiras. ${ }^{15} \mathrm{O} \mathrm{VEF}_{1} / \mathrm{CVF}$ e o percentual do previsto da CVF (ppCVF) foram considerados as principais exposições primárias como variáveis contínuas na análise principal. Em análise de sensibilidade, também analisamos os tercis de cada padrão espirométrico e, ainda, a dicotomização em padrões obstrutivo e não obstrutivo $\left(\mathrm{VEF}_{1} / \mathrm{CVF} \leq 70 \mathrm{e}\right.$ $>70$, respectivamente) e padrões restritivos e não restritivos (ppCVF $<80 \%$ e $\geq 80 \%$, respectivamente). A força muscular inspiratória (PImáx) e expiratória (PEmáx) máximas foram medidas de acordo com as recomendações, ${ }^{16}$ obtidas com 
um transdutor digital (MVD300, Globalmed, Porto Alegre, Brasil). Os participantes estavam na posição sentada usando clipe nasal e bocal. A PImáx foi determinada com um esforço inspiratório máximo a partir do volume mais próximo possível ao residual, contra uma via aérea ocluída, com escape aéreo mínimo (2mm). A PEmáx foi determinada com um esforço expiratório máximo a partir do mais próximo possível da capacidade, contra uma via aérea obstruída. Foram realizadas três a cinco manobras reprodutíveis ( $\leq 10 \%$ da variação entre os valores), sustentadas por pelo menos 1 segundo cada; foram separadas por 1 minuto de descanso e o maior valor foi utilizado para análise. ${ }^{16} \mathrm{~A}$ PImáx baixa foi considerada quando a PImáx era $\leq 80 \mathrm{cmH}_{2} \mathrm{O}$ em homens e $\leq 60 \mathrm{cmH}_{2} \mathrm{O}$ em mulheres. ${ }^{17}$

\section{Teste de esforço cardiopulmonar}

Os pacientes foram submetidos a um CPX máximo, sintoma-limitado, ${ }^{18}$ usando o protocolo de rampa com cicloergômetro (Corival, Lode, Holanda) e um analisador metabólico de gases expirados (Quark CPET, Cosmed, Itália). Calibração de gases e volumes foi realizada antes de cada teste. A ventilação-minuto (VE), o consumo de oxigênio $\left(\mathrm{VO}_{2}\right)$ e a produção de dióxido de carbono $\left(\mathrm{VCO}_{2}\right)$ foram adquiridos respiração por respiração e a média foi calculada em intervalos de 10 segundos. O limiar anaeróbio ventilatório (LAV) foi determinado pelo método $\mathrm{V}$-slope. $\mathrm{O} \mathrm{VO}_{2}$ de pico foi definido como a maior média de 10 segundos durante o platô final, se o paciente o atingiu, ou a maior média de 20 segundos no minuto final do teste sintoma-limitado. O VE/ $/ \mathrm{CO}_{2}$-slope foi calculado a partir de uma regressão linear, entre a $\mathrm{VE}$ e a $\mathrm{VCO}_{2}$ desde o início do teste até o pico do exercício. Devido à fadiga relatada nos testes preliminares de VVM (ventilação voluntária máxima) (não exibidos), subestimando resultados de manobras forçadas subsequentes ou limitando a realização de medida reprodutível, especialmente nos pacientes mais avançados, não conseguimos utilizar a VVM como padrão em toda a coorte, para garantir a comparabilidade. Portanto, a reserva ventilatória foi estimada a partir do $\mathrm{VEF}_{1}$ (calculada como 100$\left.\left[\mathrm{VE} /\left(\mathrm{VEF}_{1 \times} 40\right) \times 100\right]\right) . .^{18} \mathrm{~A}$ potência circulatória foi calculada a partir do produto do $\mathrm{VO}_{2}$ pico e pressão arterial sistólica máxima e a potência ventilatória calculada pelo quociente entre a pressão arterial sistólica máxima e a Ve/ $\mathrm{VCO}_{2}{ }_{2}$-slope. ${ }^{19}$

\section{Eventos incidentais}

O desfecho incidental foi composto de mortalidade cardiovascular, transplante cardíaco de urgência ou implantação de DAVE após a inclusão no estudo. A vigilância dos eventos ocorreu a cada 3 meses, por meio telefônico, revisando prontuários hospitalares ou confirmação de registros de atestados de óbito.

\section{Abordagem estatística}

As características gerais foram descritas usando média e desvio padrão para variáveis contínuas e números absolutos e porcentagens para variáveis categóricas. Kolmogorov-Smirnov foi utilizado e todas as variáveis mostraram distribuição normal. Para a análise transversal, foi utilizada regressão linear para avaliar associações entre $\mathrm{VEF}_{1} / \mathrm{CVF}$ e ppCVF, como variáveis de exposição contínuas, com estrutura cardíaca, força respiratória e parâmetros do CPX como variáveis dependentes, em modelos não ajustados e ajustados por idade e sexo, exibidos como coeficiente- $ß$ e intervalo de confiança $95 \%(95 \% \mathrm{IC})$, para cada 10 pontos percentuais de aumento no parâmetro espirométrico. Considerando a distribuição normal e a independência das observações dentro de cada modelo (correlação de Pearson <0,35 entre cada exposição e desfecho), as premissas foram verificadas para a regressão linear. Para abordar potenciais associações não lineares, também testamos modelos polinomiais (splines) cúbicos restritos usando 3 a 7 nós, não ajustados e ajustados para idade e sexo.

Para análise de sensibilidade, as variáveis de exposição também foram categorizadas em: a) tercis sexo-específicos para $V E F_{1} / C V F$ e ppCVF, sendo o primeiro tercil representando a pior função pulmonar, e o terceiro tercil, a melhor função pulmonar; e b) grupos dicotômicos, comparando padrões obstrutivos (VEF $\mathrm{V}_{1} / \mathrm{CVF}$ $\leq 70)$ com não obstrutivos $\left(V^{2} F_{1} / C V F>70\right)$ e padrões restritivos ( $p$ pCVF <80\%) com não restritivos ( $p p C V F$ $\geq 80 \%$ ). Regressões lineares e logísticas e teste de Quiquadrado para tendência foram usados para avaliar as associações. Para verificar possíveis assimetrias entre participantes incluídos e excluídos, esses grupos foram comparados usando o Qui-quadrado para variáveis categóricas e teste $t$ para amostras independentes para contínuas.

Para a análise prospectiva, regressão de Cox foi utilizada para determinar a magnitude da associação entre a redução de 10 pontos percentuais na função pulmonar com o a incidência do desfecho composto, mostrado como hazard ratio (HR) e IC 95\%. Associações não lineares foram investigadas usando regressão cúbica restrita (spline) com o número de nós selecionados para minimizar o modelo AIC (3 a 7 nós testados). A presunção de riscos proporcionais foi testada para todos os modelos usando resíduos de Schoenfeld, e nenhuma violação foi detectada.

Para a análise prospectiva, a regressão de Cox foi usada para determinar a magnitude da associação da diminuição de 10 pontos percentuais na variável da espirometria com o desfecho composto incidental, mostrado como razão de risco (HR) e IC 95\%. As associações não lineares foram investigadas usando regressão spline cúbica restrita com o número de nós selecionados para minimizar o modelo AIC (3 a 7 nós testados). A suposição de riscos proporcionais foi testada para todos os modelos usando resíduos de Schoenfeld, e nenhuma violação foi detectada. Como abordagem de sensibilidade, regressões de Cox foram realizadas restringindo 4 subgrupos: FEVE $\leq 50 \%$; FEVE $>50 \%$; PImáx baixa; e PImáx normal.

Um valor de $p$ bicaudal $<0,05$ foi considerado significativo para todas as análises. Foi utilizado o programa STATA versão 14.2 (Stata Corp LP, College Station, Texas, EUA).

\section{Resultados}

Entre os 111 participantes com IC, a etiologia isquêmica foi predominante, estágios American Heart Association C 
ou $\mathrm{D}$, tratados de acordo com as diretrizes, dentre os quais 24 apresentaram FEVE $>50 \%$ (Tabela1). Aproximadamente metade dos indivíduos apresentava um padrão restritivo (ppCVF <80\%); um quarto, um padrão obstrutivo $\left(\mathrm{VEF}_{1} /\right.$ CVF $\leq 70)$; e 14 indivíduos (13\%), disfunções combinadas; enquanto, em 40 deles (36\%), a espirometria era normal. Dos 26 (23\%) pacientes com índice de massa corporal (IMC) maior que $30 \mathrm{~kg} / \mathrm{m}^{2}$, 15 (65\%) apresentaram um ppCVF <80\%. Entre 57 pacientes com ppCVF $<80 \%$ (51\%), 15 tinham IMC $>30 \mathrm{~kg} / \mathrm{m}^{2}$. Baixa PImáx foi um achado frequente. $\mathrm{O}$ $\mathrm{VO}_{2}$ de pico médio foi baixo, mesmo garantindo critérios de esforço máximo. A fadiga muscular geral ou a de membros inferiores foram sintomas limitantes, não houve sibilância ou cianose. Cinco pacientes apresentaram reserva ventilatória inferior a $20 \%$, dentre eles 4 com $10 \%$ a $15 \%$; com distúrbios basais restritivos (3) ou combinados (2) e FEVE <34\%. Entre estes, o intervalo RQ foi 1,09 e 1,22. Aqueles não incluídos por falta de dados ou por espirometria de má qualidade apresentaram características semelhantes aos incluídos, exceto pela média de idade mais jovem $(51,6 \pm 14,2$ anos) (Tabela Suplementar S1).

\section{Relação do $\mathrm{VEF}_{1} / \mathrm{CVF}$ com variáveis funcionais e com prognóstico}

Em modelos contínuos, o VEF $/$ CVF foi proporcionalmente associado ao $\mathrm{VEF}_{1}$ e à reserva ventilatória pelo $\mathrm{CPX}$, de modo que, a cada aumento de 10 pontos percentuais no $\mathrm{VEF}_{1} / \mathrm{CVF}$, houve aumento de $200 \mathrm{~mL}$ (IC 95\% 100-310mL, $\mathrm{p}<0,001$ ) em $\mathrm{VEF}_{1}$ e de 7 pontos percentuais (IC 95\% $3-10 \%$; $p<0,001)$ de aumento na reserva ventilatória, após ajuste para idade e sexo (Tabela 2). Embora a PImáx baixa fosse um achado comum em indivíduos com padrão obstrutivo ( $n=15,54 \%)$, a frequência foi semelhante quando comparada ao padrão não obstrutivo $(n=36$, $47 \%$; $p=0,54)$, e a PImáx não foi associada ao $V_{E F} /$ CVF contínuo $(p=0,90)$. Além disso, foi observada uma associação não linear entre $\mathrm{VEF}_{1} / \mathrm{CVF}$ e $\mathrm{VEF}_{1}$, de forma que essa relação é mais robusta se $\mathrm{VEF}_{1} / \mathrm{CVF}$ for inferior a $75 \%$ (Figura 1A). Nenhuma outra métrica de estrutura ou função cardiopulmonar foi associada com VEF ${ }_{1} / C V F$. Esses achados foram consistentes também entre os tercis $V E F_{1} /$ CVF (Tabela Suplementar S2).

No seguimento médio de 2,2 $\pm 0,7$ anos, 15 indivíduos tiveram morte cardiovascular, 3 foram transplantados e 4 tiveram implante de DAVE. Houve tendência de menor $V E F_{1} / C V F$ aumentar o risco para o desfecho composto, porém não foi linearmente significativo quando considerados a idade e o sexo (Tabela 3). Por outro lado, houve associação não linear entre $\mathrm{VEF}_{1} / \mathrm{CVF}$ e o desfecho composto, de forma que o risco diminui quando $\mathrm{VEF}_{1} / \mathrm{CVF}$ $>75$ (Figura 2).

Duas análises de sensibilidade foram realizadas. Primeiramente, excluindo aqueles com FEVE $>50 \%$ $(n=24)$, dentre os 87 sujeitos restantes, 20 eventos ocorreram. Nesse cenário, cada redução de 10 pontos percentuais no $\mathrm{VEF}_{1} / \mathrm{CVF}$ foi associada a um aumento de $50 \%$ na probabilidade de o desfecho composto incidir por ano de observação, levando em consideração a idade e o sexo $(p=0,04)$ (Figura Suplementar S1). Entre aqueles com FEVE $>50 \%$, apenas dois eventos ocorreram. Em segundo lugar, entre indivíduos com baixa PImáx $(n=51$, 13 eventos), $\mathrm{VEF}_{1} / \mathrm{CVF}$ reduzido foi associado a maior risco para o desfecho primário (HR 1,72; 1,14-2,61; $p=0,009)$, enquanto no subgrupo com PImáx normal $(n=57$, seis eventos), não foi associado ao desfecho (HR 0,98; 0,36-2,69) (Figura Suplementar S1)

\section{Relação da porcentagem da CVF prevista com variáveis funcionais e com prognóstico}

Considerando a idade e o sexo, cada aumento de 10 pontos percentuais no ppCVF ajustado foi proporcionalmente associado a um aumento linear no $\mathrm{VEF}_{1^{\prime}}$ de 230mL (IC 95\% 190-270mL, p <0,001) (Tabela 2). A PImáx também aumentou 3,8 $\mathrm{cmH}_{2} \mathrm{O}(\mathrm{IC} 95 \%$ 0,3-7,3, p = $0,03)$, mas a análise não linear mostrou que essa associação foi mais robusta para ppCVF $<80 \%$ (Tabela 2 e Figura $1 \mathrm{G}$ ). A PImáx baixa foi mais frequente em indivíduos com IC com padrão restritivo $(n=34,65 \%)$ quando comparados àqueles sem padrão restritivo $(n=17,32 \%$; $p<0,001)$. A FEVE aumentou aproximadamente 2 pontos percentuais para cada aumento de 10 pontos percentuais no ppCVF, que também foi mais proeminente quando ppCVF $<80 \%$ (Figura 1F). Em relação ao CPX, quanto maior o ppCVF, maior a potência de pico, o $\mathrm{VO}_{2}$ de pico relativo e a reserva ventilatória nos modelos ajustados. Nenhuma outra métrica de estrutura ou função cardiopulmonar foi associada ao ppCVF, como variável contínua (Tabela 2) ou categorizada em tercis (Tabela Suplementar S3).

O ppFVC mais baixo não foi capaz de distinguir indivíduos com IC sob maior risco para o desfecho composto na análise primária (Tabela 3 e Figura 2) ou de sensibilidade (Figura Suplementar S2).

\section{Discussão}

Em uma coorte de mundo real de 111 indivíduos com IC crônica de classes C ou D, dentro de uma ampla faixa de fração de ejeção, investigamos como o espectro de distúrbios espirométricos normal a grave se relacionava com as métricas funcionais de repouso e de exercício, e com eventos cardiovasculares incidentais maiores. Em todas as faixas de obstrução das vias aéreas e de comprometimento da capacidade vital, levando em consideração a idade e o sexo, tanto o $\mathrm{VEF}_{1} / \mathrm{CVF}$ baixo quanto o ppCVF foram associados à redução da reserva ventilatória ao exercício, mas apenas o ppCVF baixo foi associado à menor fração de ejeção, fraqueza inspiratória e menor capacidade de exercício, que foi mais proeminente quando o ppCVF foi inferior a $80 \%$. Embora tais disfunções pulmonares fossem comuns, o risco para o desfecho composto de morte cardiovascular, transplante cardíaco ou implante de DAVE teve associação não linear apenas com $V F_{1} / C V F$, mas não com ppCVF, sugerindo um melhor prognóstico com padrão não obstrutivo $\left(V F_{1} / C V F>75 \%\right)$. Além disso, entre os subgrupos de baixa FEVE e baixa PImáx, apenas o VEF $/ C V F$ reduzido distinguiu um risco maior. Portanto, $V_{E F} / C V F$ e ppCVF fenotipam aspectos clínicos de pacientes com IC de maneira diferente (Figura 3). 
Tabela 1 - Características basais da população com insuficiência cardíaca $(n=111)$. Valores são mostrados como média \pm DP ou n $(\%)$

\begin{tabular}{|c|c|}
\hline Participantes, $\mathrm{n}$ & 111 \\
\hline \multicolumn{2}{|l|}{ Dados demográficos e clínicos } \\
\hline Idade, anos & $57,4 \pm 11,8$ \\
\hline Masculino, n(\%) & $67(60 \%)$ \\
\hline \multicolumn{2}{|l|}{ Etiologia, $\mathrm{n}(\%)$} \\
\hline Chagas & $32(29 \%)$ \\
\hline Isquêmica & $43(39 \%)$ \\
\hline Idiopática & $23(21 \%)$ \\
\hline Outra & $13(12 \%)$ \\
\hline $\mathrm{IMC}, \mathrm{kg} / \mathrm{m}^{2}$ & $26,6 \pm 4,8$ \\
\hline $\mathrm{IMC}>30$ kg/m²; $\mathrm{n}(\%)$ & $26(23 \%)$ \\
\hline \multicolumn{2}{|l|}{ História médica } \\
\hline Hipertensão, n(\%) & $63(57 \%)$ \\
\hline Diabetes, $\mathrm{n}(\%)$ & $20(18 \%)$ \\
\hline Fumantes ativos, $\mathrm{n}(\%)$ & $29(26 \%)$ \\
\hline Dislipidemia, $\mathrm{n}(\%)$ & $44(40 \%)$ \\
\hline \multicolumn{2}{|l|}{ NYHA, $n(\%)$} \\
\hline I & $15(13 \%)$ \\
\hline II & $25(22 \%)$ \\
\hline III & $71(64 \%)$ \\
\hline \multicolumn{2}{|l|}{ Medicações e dispositivos } \\
\hline Betabloqueadores, $\mathrm{n}(\%)$ & $100(90 \%)$ \\
\hline IECA/BRA, $n(\%)$ & $94(84 \%)$ \\
\hline Espironolactona, $\mathrm{n}(\%)$ & $73(66 \%)$ \\
\hline Digoxina, $\mathrm{n}(\%)$ & $22(20 \%)$ \\
\hline Estatina, $\mathrm{n}(\%)$ & $70(63 \%)$ \\
\hline Furosemida, $\mathrm{n}(\%)$ & $67(60 \%)$ \\
\hline Marca-passo/CDI, n(\%) & $25(22 \%)$ \\
\hline \multicolumn{2}{|l|}{ Função pulmonar } \\
\hline $\mathrm{VEF}_{1}, \mathrm{~L}$ & $2.3 \pm 0.7$ \\
\hline CVF, L & $3.0 \pm 0.9$ \\
\hline Porcentagem prevista da CVF, \% & $80 \pm 17$ \\
\hline Porcentagem prevista da CVF $<80 \%$ & $57(51 \%)$ \\
\hline $\mathrm{VEF}_{1} / \mathrm{CVF}$ & $75 \pm 9$ \\
\hline $\mathrm{VEF}_{1} / \mathrm{CVF} \leq 70$ & $28(25 \%)$ \\
\hline PEmáx, $\mathrm{cmH}_{2} \mathrm{O}$ & $84,7 \pm 40,1$ \\
\hline Plmáx, $\mathrm{cmH}_{2} \mathrm{O}$ & $75,4 \pm 35,4$ \\
\hline PImáx reduzida, $\mathrm{n}(\%)$ & $51(49 \%)$ \\
\hline \multicolumn{2}{|l|}{ Ecocardiograma } \\
\hline FEVE, \% & $38,4 \pm 15,0$ \\
\hline FEVE $>50 \%, n(\%)$ & $24(23 \%)$ \\
\hline Volume do $\mathrm{AE}$ indexado, $\mathrm{mL} / \mathrm{m}^{2}$ & $44,7 \pm 16,7$ \\
\hline PSAP estimada, $\mathrm{mmHg}$ & $38,9 \pm 12,0$ \\
\hline \multicolumn{2}{|l|}{ Teste cardiopulmonar } \\
\hline Potência de pico, W & $80,3 \pm 30,6$ \\
\hline Frequência cardíaca de pico, bpm & $118 \pm 26$ \\
\hline Pressão sistólica de pico, mmHg & $151 \pm 25$ \\
\hline $\mathrm{VO}_{2}$ absoluto de pico, $\mathrm{mL} / \mathrm{min}$ & $966 \pm 401$ \\
\hline $\mathrm{VO}_{2}$ relativo de pico, $\mathrm{mL} / \mathrm{kg} / \mathrm{min}$ & $13,4 \pm 4,6$ \\
\hline RER & $1,23 \pm 0,18$ \\
\hline $\mathrm{VO}_{2}$ absoluto no LAV, $\mathrm{mL} / \mathrm{min}$ & $618 \pm 281$ \\
\hline $\mathrm{VO}_{2}$ relativo no $\mathrm{LAV}, \mathrm{mL} / \mathrm{kg} / \mathrm{min}$ & $8,6 \pm 3,5$ \\
\hline Pulso de $\mathrm{O}_{2}, \mathrm{~mL} /$ batimento & $8.4 \pm 3.1$ \\
\hline OUES & $1145 \pm 465$ \\
\hline VE max, (L/min) & $45.0 \pm 16.6$ \\
\hline Reserva ventilatória, $\%$ & $48 \pm 19$ \\
\hline VENCCO ${ }_{2}$ slope & $37.3 \pm 8.1$ \\
\hline Poder circulatório, $\mathrm{mmHg} \cdot \mathrm{mL} / \mathrm{kg} / \mathrm{min}$ & $2165 \pm 1024$ \\
\hline Poder ventilatório, $\mathrm{mmHg}$ & $4.3 \pm 1.4$ \\
\hline
\end{tabular}

IMC: índice de massa corporal; VEF : volume expirado forçado em 1 segundo; CVF: capacidade vital forçada; NYHA: classe funcional da New York Heart Association; IECA: inibidores da enzima de conversão da angiotensina; BRA: bloqueadores do receptor da angiotensina II; CDI: cardioversor desfibrilador implantável; PEmáx: pressão expiratória máxima; PImáx: pressão inspiratória máxima; FEVE: fração de ejeção do ventrículo esquerdo do ventrículo esquerdo; AE: átrio esquerdo; PSAP: pressão sistólica da artéria pulmonar; $V_{2}$ : consumo de oxigênio; OUES: curva da eficiência de captação de oxigênio; VE: ventilação minuto; VENCO ${ }_{2}$ slope: VE/produção de dióxido de carbono; RER: razão de troca respiratória; LAV: limiar anaeróbio ventilatório. 
Tabela 2 - Relação contínua entre os parâmetros espirométricos (por aumento de 10 pontos percentuais em cada VEF/CVF e ppCVF) e a função cardiopulmonar em participantes com insuficiência cardíaca

\begin{tabular}{|c|c|c|c|c|c|}
\hline \multirow{2}{*}{ Função cardíaca e pulmonar } & & \multicolumn{2}{|c|}{$\operatorname{VEF}_{1} / \mathrm{CVF}$} & \multicolumn{2}{|c|}{$\%$ prevista da CVF } \\
\hline & & Coeficiente (95\%IC) & $\mathbf{p}$ & Coeficiente $(95 \% \mathrm{IC})$ & p \\
\hline \multirow{2}{*}{$\begin{array}{l}\text { VEF }_{1} \\
L\end{array}$} & Modelo 1 & $0,24(0,10 ; 0,38)$ & 0,001 & $0,25(0,18 ; 0,31)$ & $<0,001$ \\
\hline & Modelo 2 & $0,20(0,10 ; 0,31)$ & $<0,001$ & $0,23(0,19 ; 0,27)$ & $<0,001$ \\
\hline \multirow{2}{*}{$\begin{array}{l}\text { PEmáx } \\
\mathrm{CmH}_{2} \mathrm{O}\end{array}$} & Modelo 1 & $2,7(-6,6 ; 11,9)$ & 0,57 & $-0,9(-5,8 ; 4,1)$ & 0,73 \\
\hline & Modelo 2 & $0,6(-7,5 ; 8,8$ & 0,88 & $-1,5(-5,8 ; 2,9)$ & 0,50 \\
\hline \multirow{2}{*}{$\begin{array}{l}\text { PImáx, } \\
\mathrm{CmH}_{2} \mathrm{O}\end{array}$} & Modelo 1 & $0,9(-0,6 ; 8,2)$ & 0,81 & $4,1(0,2 ; 8,1)$ & 0,04 \\
\hline & Modelo 2 & $0,4(-6,1 ; 6,9)$ & 0,90 & $3,8(0,3 ; 7,3)$ & 0,031 \\
\hline \multirow{2}{*}{ FEVE, \% } & Modelo 1 & $1,9(-1,0 ; 4,9)$ & 0,20 & $2,2(0,6 ; 3,9)$ & 0,007 \\
\hline & Modelo 2 & $1,6(-1,4 ; 4,7)$ & 0,28 & $2,1(0,5 ; 3,8)$ & 0,013 \\
\hline \multirow{2}{*}{$\begin{array}{l}\text { Potência pico, } \\
\text { W }\end{array}$} & Modelo 1 & $1,5(-4,6 ; 7,6)$ & 0,63 & $4,2(0,9 ; 7,5)$ & 0,012 \\
\hline & Modelo 2 & $0,3(-4,3 ; 4,9)$ & 0,89 & $3,5(1,1 ; 6,0)$ & 0,005 \\
\hline \multirow{2}{*}{$\mathrm{VO}_{2}$ absoluto de pico, $\mathrm{mL} / \mathrm{min}$} & Modelo 1 & $30(-50 ; 111)$ & 0,45 & $35(-9 ; 78)$ & 0,11 \\
\hline & Modelo 2 & $18(-49 ; 86)$ & 0,59 & $27(-9 ; 63)$ & 0,14 \\
\hline \multirow{2}{*}{$\mathrm{VO}_{2}$ relativo de pico, $\mathrm{mL} / \mathrm{kg} / \mathrm{min}$} & Modelo 1 & $-0,3(-1,3 ; 0,6)$ & 0,47 & $0,6(0,1 ; 1,1)$ & 0,02 \\
\hline & Modelo 2 & $-0,4(-1,3 ; 0,4)$ & 0,30 & $0,5(0,1 ; 1,0)$ & 0.028 \\
\hline \multirow{2}{*}{ Razão de troca ventilatória } & Modelo 1 & $-0,01(-0,05 ; 0,02)$ & 0,48 & $0,02(-0,003 ; 0,04)$ & 0,09 \\
\hline & Modelo 2 & $-0,01(-0,05 ; 0,02)$ & 0,40 & $0,01(-0,003 ; 0,03)$ & 0,10 \\
\hline \multirow{2}{*}{$\mathrm{VO}_{2}$ absoluto no LAV, $\mathrm{mL} / \mathrm{min}$} & Modelo 1 & $-8(-66 ; 49)$ & 0,77 & $12(-20 ; 44)$ & 0,45 \\
\hline & Modelo 2 & $-12(-67 ; 44)$ & 0,68 & $13(-18 ; 44)$ & 0,42 \\
\hline \multirow{2}{*}{$\mathrm{VO}_{2}$ relativo no $\mathrm{LAV}, \mathrm{mL} / \mathrm{kg} / \mathrm{min}$} & Modelo 1 & $-0,7(-1,4 ;-0,004)$ & 0,05 & $0,2(-0,2 ; 0,6)$ & 0,31 \\
\hline & Modelo 2 & $-0,7(-1,4 ; 0,007)$ & 0,05 & $0,2(-0,2 ; 0,6)$ & 0,25 \\
\hline \multirow{2}{*}{$\begin{array}{l}\text { Pulso de } \mathrm{O}_{2} \\
\mathrm{~mL} / \text { batimento }\end{array}$} & Modelo 1 & $0,4(-0,2 ; 1,0)$ & 0,21 & $0,1(-0,2 ; 0,5)$ & 0,45 \\
\hline & Modelo 2 & $0,4(-0,2 ; 0,9)$ & 0,18 & $0,1(0,2 ; 0,4)$ & 0,47 \\
\hline \multirow{2}{*}{ OUES } & Modelo 1 & $62(-31 ; 155)$ & 0,19 & $19(-32 ; 70)$ & 0,47 \\
\hline & Modelo 2 & $48(-34 ; 130)$ & 0,25 & $9(-36 ; 54)$ & 0,70 \\
\hline \multirow{2}{*}{$\begin{array}{l}\text { VE máx, } \\
\text { L/min }\end{array}$} & Modelo 1 & $-0,4(-3,7 ; 2,9)$ & 0,82 & $1,6(-0,2 ; 3,4)$ & 0,08 \\
\hline & Modelo 2 & $-0,6(-3,3 ; 2,1)$ & 0,68 & $1,5(0,02 ; 2,9)$ & 0,05 \\
\hline \multirow{2}{*}{ Reserva ventilatória, \% } & Modelo 1 & $7,4(3,9 ; 10,9)$ & $<0,001$ & $4,6(2,8 ; 6,5)$ & $<0,001$ \\
\hline & Modelo 2 & $6,8(3,3 ; 10,3)$ & $<0,001$ & $4,3(2,5 ; 6,2)$ & $<0,001$ \\
\hline \multirow{2}{*}{$\mathrm{VE} / \mathrm{NCO}_{2}$ slope } & Modelo 1 & $-0,2(-2,0 ; 1,7)$ & 0,87 & $-0,6(-1,6 ; 0,3)$ & 0,19 \\
\hline & Modelo 2 & $0,2(-1,6 ; 2,0)$ & 0,85 & $-0,5(-1,5 ; 0,5)$ & 0,35 \\
\hline \multirow{2}{*}{ Pot. circulatória, $\mathrm{mmHg} \cdot \mathrm{mL} / \mathrm{kg} / \mathrm{min}$} & Modelo 1 & $-20(-202 ; 163)$ & 0,83 & $85(-14 ; 183)$ & 0,09 \\
\hline & Modelo 2 & $-43(-212 ; 125)$ & 0,61 & $72(-19 ; 163)$ & 0,12 \\
\hline \multirow{2}{*}{ Pot. ventilatória, $\mathrm{mmHg}$} & Modelo 1 & $0,08(-0,20 ; 0,35)$ & 0.59 & $0.12(-0,03 ; 0,27)$ & 0,11 \\
\hline & Modelo 2 & $0,04(-0,22 ; 0,31)$ & 0.76 & $0.10(-0,04 ; 0,24)$ & 0,17 \\
\hline
\end{tabular}

VEF : volume expirado forçado em 1 segundo; CVF: capacidade vital forçada; PEmáx: pressão expiratória máxima; PImáx: pressão inspiratória máxima; FEVE: fração de ejeção do ventrículo esquerdo do VE; $\mathrm{VO}_{2}$ : consumo de oxigênio; LAV: limiar anaeróbio ventilatório; OUES: curva da eficiência de captação de oxigênio; VE: ventilação minuto; $\mathrm{VCO}_{2}$ : produçã̃o de dióxido de carbono; Pot.: potência. Modelo 1: não ajustado; Modelo 2: idade e sexo. Nota: valores de $p$ se referem à respectiva análise de regressão linear. 


\section{Artigo Original}

A

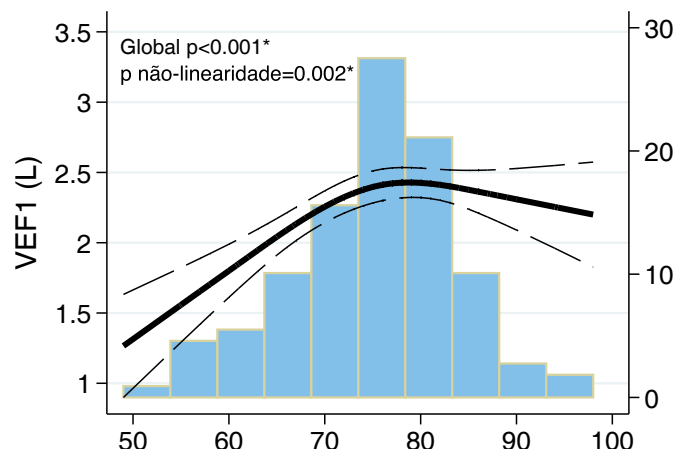

B
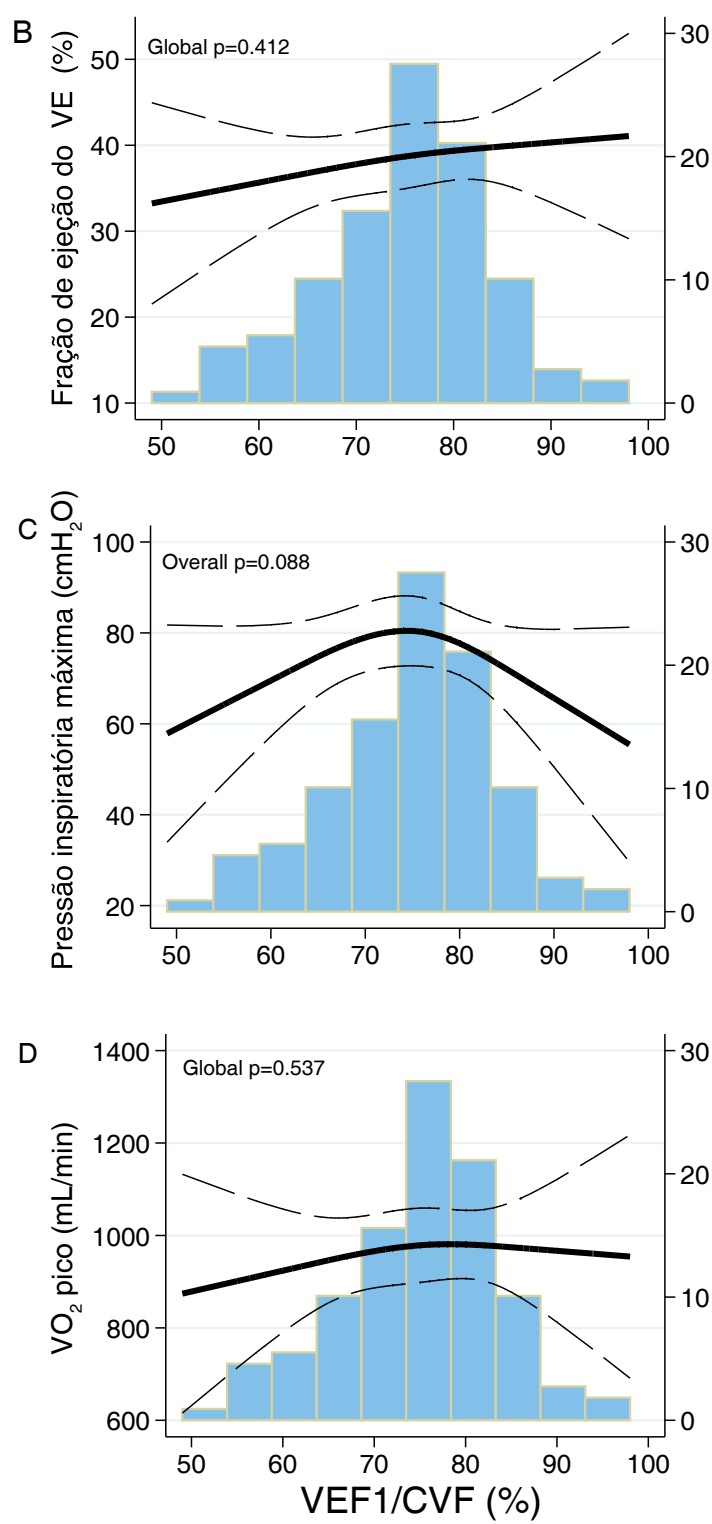
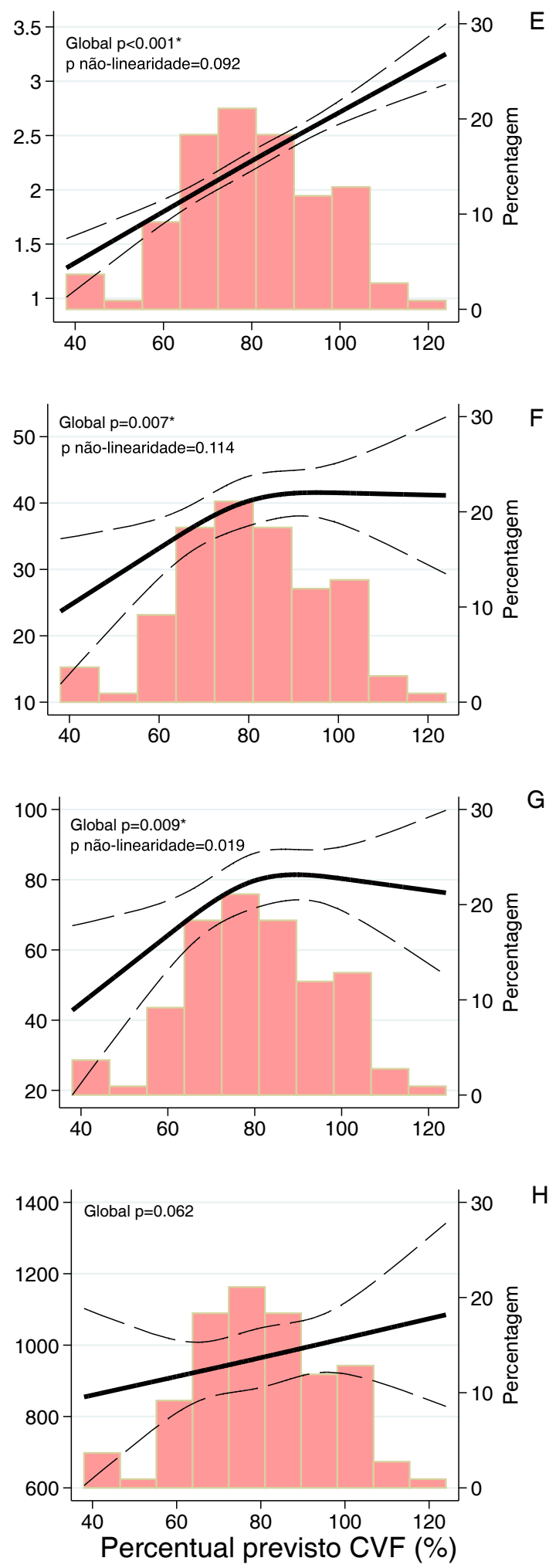

Figura 1 - Associação contínua da VEF /CVF (azul) e do percentual previsto da CVF (vermelho-claro) com VEF , FEVE, PImáx e VO de usando splines cúbicos restritos. Modelos foram construídos usando splines cúbicos restritos com 3 nós. ${ }^{*} p<0,05$ em modelos adicionalmente ajustados para idade e sexo. 
Tabela 3 - Associação das variáveis espirométricas basais com a incidência do desfecho composto (mortalidade cardiovascular, transplante cardíaco e implante de dispositivo de assistência ventricular esquerda; 22 eventos) entre participantes com insuficiência cardíaca ( $n=111)$, acompanhados por $2,2 \pm 0,7$ anos

\begin{tabular}{|c|c|c|c|c|c|c|}
\hline & $\mathbf{n}$ & Eventos & $\begin{array}{l}\text { Não ajustado } \\
\text { HR }(95 \% \text { IC })^{*}\end{array}$ & $\mathbf{p}$ & $\begin{array}{c}\text { Ajuste por sexo } \\
\text { e idade } \\
\text { HR }(95 \% I C) *\end{array}$ & $\mathbf{p}$ \\
\hline \multicolumn{7}{|l|}{$\mathrm{VEF}_{1} / \mathrm{CVF}$} \\
\hline Obstrutivo & 28 & 9 & \multirow{2}{*}{$2,45(1,05-5,77)$} & \multirow{2}{*}{$p=0,039$} & \multirow{2}{*}{$2,28(0,95-5,44)$} & \multirow{2}{*}{$p=0,064$} \\
\hline Não obstrutivo & 83 & 13 & & & & \\
\hline Contínuo & 111 & 22 & $1,48(1,00-2,18)$ & $p=0,050$ & $1,44(0,97-2,13)$ & $p=0,069$ \\
\hline \multicolumn{7}{|l|}{ ppCVF } \\
\hline Restritivo & 57 & 14 & \multirow{2}{*}{$1,83(0,77-4,37)$} & \multirow{2}{*}{$p=0,172$} & \multirow{2}{*}{$1,86(0,78-4,44)$} & \multirow{2}{*}{$p=0,163$} \\
\hline Não restritivo & 54 & 8 & & & & \\
\hline Contínuo & 111 & 22 & $1,16(0,92-1,46)$ & $p=0,207$ & $1,13(0,89-1,43)$ & $p=0,306$ \\
\hline
\end{tabular}

*por 10 unidades de redução. Nota: valores de $p$ se referem à respectiva análise de regressão de Cox.

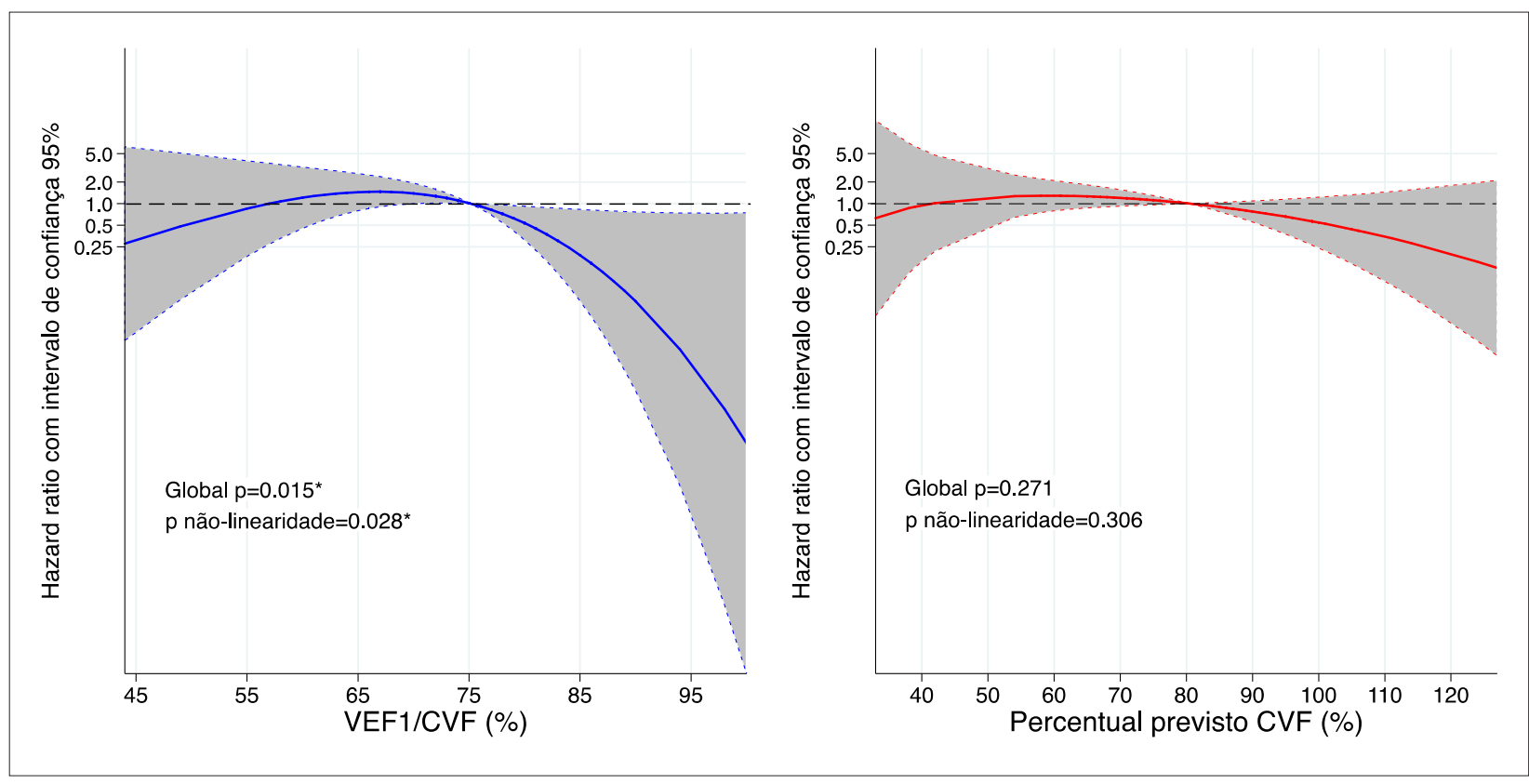

Figura 2 - Associações contínuas de VEF /CVF (azul) e percentual previsto da CVF (vermelho) basais com o desfecho composto (morte cardiovascular, transplante cardíaco e implante de dispositivo de assistência ventricular), em tempo médio de acompanhamento de 2,2士0,7 anos (22 eventos). Modelos foram construídos para a variável primária de exposição (VEF/CVF e percentual previsto da CVF) usando splines cúbicos restritos com 3 nós. Linear corresponde à análise de regressão de Cox. ${ }^{*} p<0,05$ em modelos adicionalmente ajustados para idade e sexo.

Relação de função pulmonar de repouso com desempenho ao exercício

Os efeitos diretos da IC nas vias aéreas, como congestão vascular, edema parenquimatoso e alveolar e fibrose intersticial, estão relacionados à constrição aguda/subaguda do diâmetro das vias aéreas e à redução subaguda/crônica do volume pulmonar. ${ }^{1-3}$ Como resultado, o VEF 1 e a CVF diminuem de forma independente ou paralela, sugerindo que mesmo a disfunção subclínica pode contribuir de forma diferente para a intolerância ao exercício. Consequentemente, o $\mathrm{VEF}_{1}$ se associa de forma mais robusta à capacidade aeróbia
$\left(\mathrm{VO}_{2}\right.$ de pico) do que a FEVE. ${ }^{8,20}$ Portanto, espera-se que a ventilação voluntária máxima (derivada do $V_{E F}$ ) seja prejudicada na $\mathrm{IC}$, proporcionalmente à gravidade da disfunção pulmonar subjacente. No entanto, a intolerância ao exercício em indivíduos com IC é multifatorial, e a reserva ventilatória reduzida no pico expressa apenas parcialmente a contribuição pulmonar. ${ }^{7}$ De fato, observamos que a reserva ventilatória aumentou 7 e 4 pontos percentuais para cada aumento de 10 pontos percentuais no VEF /CVF e na ppFVC, respectivamente. No entanto, a reserva ventilatória foi tão baixa quanto $38 \%$ e $39 \%$, em média, nos tercis inferiores de 


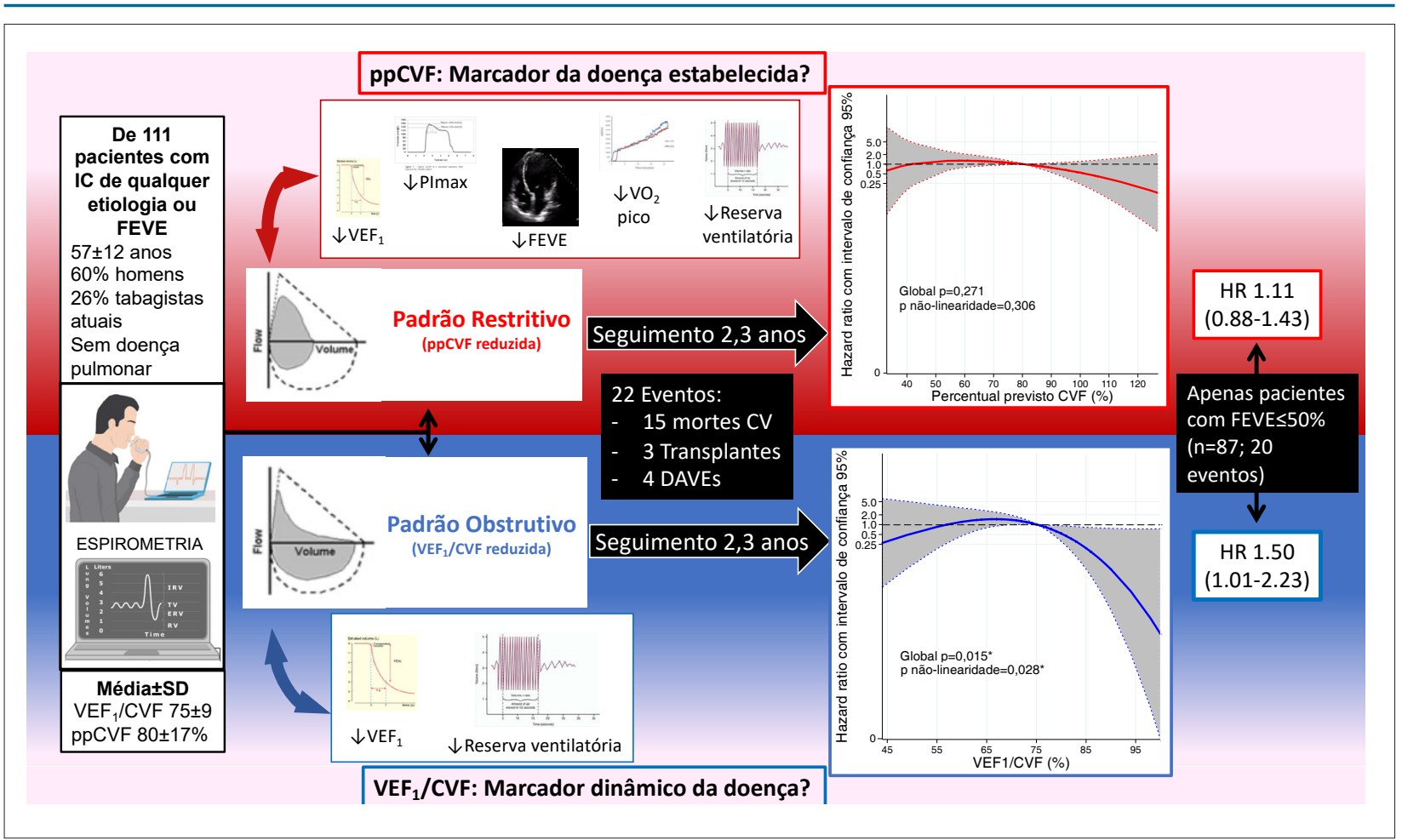

Figura 3 - Resumo visual dos achados principais: VEF,/CVF e ppCVF caracterizam de forma diferencial pacientes com insuficiência cardiaca. IC: insuficiência cardiaca; FEVE: fração de ejeção do ventrículo esquerdo; VEF : volume expiratório forçado em 1 segundo; ppCVF: percentual previsto da capacidade vital forçada; PImáx: pressão inspiratória máxima; CV: cardiovascular; DAVE: dispositivo de assistência ventricular esquerda; HR: hazard ratio. Apesar de a ppCVF estar associada a outras variáveis funcionais além da reserva ventilatória, apenas o VEF/CVF foi associado a prognóstico de curto prazo, particularmente para aqueles com fração de ejeção reduzida, sugerindo que cada marcador agrega diferentes informações em relação aos fenótipos da insuficiência cardíaca.

$\mathrm{VEF}_{1} / \mathrm{CVF}$ e ppCVF, respectivamente; valores maiores que os $20 \%$ esperados para assumir inequivocamente uma restrição ventilatória no pico do exercício, sugerindo a limitação cardiocirculatória como origem primária - mas não única da limitação de esforço em nossos pacientes.

Parâmetros como OUES e VE/VCO 2 slope são menos dependentes do pico de esforço e são mais sensíveis para distinguir restrições ventilatórias de cardiocirculatórias. ${ }^{20,21}$ Os valores médios reduzidos do OUES, elevados de VE/ $\mathrm{VCO}_{2}$ slope e reduzidos do pulso de $\mathrm{O}_{2}$ em nosso estudo, na verdade, sugerem uma limitação cardiocirculatória predominante, mas a falta de associação entre $V_{E F} / C V F$ e ppCVF em repouso com outras variáveis ventilatórias de exercício, incluindo eficiência ventilatória, foi contrária à nossa hipótese a priori. Portanto, a capacidade da espirometria de repouso para determinar precisamente a contribuição ventilatória para limitação ao exercício, além da reserva ventilatória, pode ser limitada, sobrepujada pelo componente cardiocirculatório nos estágios de IC mais avançados, como em nossa coorte, composta de pacientes em estágios C/D, predominantemente em NYHA III (64\%) e $\mathrm{VO}_{2}$ pico médio de $13 \mathrm{~mL} / \mathrm{kg} / \mathrm{min}^{22}$

Em relação às outras variáveis funcionais, apenas $O$ ppCVF, mas não o $V E F_{1} / C V F$, foi adicionalmente associado à PImáx em repouso, mas não à PEmáx, à FEVE e ao pico de potência e $\mathrm{VO}_{2}$ pico relativo, levando em consideração a idade e o sexo.
Possivelmente, dadas as características graves, mas estáveis dos indivíduos com IC neste estudo, as correlações discrepantes para cada exposição poderiam resultar da influência primária da IC na redução da capacidade pulmonar total, diminuindo desproporcionalmente a CVF em relação ao $V F_{1}$, atenuando, portanto, o efeito do $\mathrm{VEF}_{1} / \mathrm{CVF}$ para prever as respostas do exercício. ${ }^{1}$ Assim, um padrão restritivo é comum na síndrome de $\mathrm{IC}_{1}^{1,7,8}$ particularmente em ICFER. ${ }^{23}$ Além disso, a relação direta do ppCVF com a FEVE apoia a hipótese de que um coração potencial aumentado e disfuncional está relacionado ao volume pulmonar reduzido devido aos efeitos de ocupação de espaço e de congestão vascular e parenquimatosa mencionados anteriormente. Tais alterações, agravadas pela fraqueza inspiratória, podem comprometer a mecânica respiratória em resposta às demandas crescentes, reduzindo ainda mais a complacência pulmonar, e todas podem contribuir para a limitação do exercício, representada pelo baixo $\mathrm{VO}_{2}$ de pico. ${ }^{1,7,8}$

Curiosamente, apenas o ppCVF foi associado a PImáx. Na IC, a redução da capacidade vital está associada à baixa PImáx e à disfunção diafragmática, ${ }^{24,25}$ que desempenha papel significativo na limitação do exercício na ICFER ${ }^{26,27}$ e na ICFEP ${ }^{28}$ e demonstra relevância prognóstica independente. ${ }^{29}$ Consistentemente com nossos achados, a disfunção e as anormalidades estruturais da musculatura esquelética generalizada, diafragmática em particular, 
contribuem amplamente para a intolerância ao exercício em ambas, ICFER e ICFEF. ${ }^{30}$ Automaticidade e constante sobrecarga de trabalho, mesmo em estado de repouso, caracterizam unicamente a predisposição do diafragma à disfunção precoce na síndrome de $\mathrm{IC}$, que é mais proeminente que a de outros músculos expiratórios e/ou periféricos. ${ }^{31}$

\section{Função pulmonar e prognóstico cardiovascular}

O declínio da função pulmonar, mesmo subclínico, mostrou-se associado à incidência de insuficiência cardíaca em populações não selecionadas. ${ }^{32,33}$ Além disso, em indivíduos com ICFER estável, a espirometria previu significativamente mortalidade por qualquer causa. ${ }^{34}$ Olson et al. ${ }^{34}$ estudaram 134 indivíduos com ICFER com $\mathrm{VO}_{2}$ pico de $19 \mathrm{~mL} / \mathrm{kg} / \mathrm{min}$ e $66 \%$ eram NYHA classes I e II, e mostraram que quanto menor VEF $_{1}$ ou CVF, menores as taxas de sobrevida. Em contraste, na ICFER avançada pré-transplante, Georgiopoulou et al. demonstraram que a espirometria não forneceu informações prognósticas. ${ }^{35}$ Em nossa coorte, posicionada entre os estudos anteriores quanto à gravidade dos participantes, o $\mathrm{VEF}_{1} /$ CVF contínuo e o ppCVF não foram capazes de distinguir indivíduos com IC com risco aumentado para eventos cardiovasculares maiores em modelos lineares.

Provavelmente, dados os estágios crônicos da IC e a limitação cardiocirculatória predominante do CPX conforme demonstrado, a contribuição do comprometimento ventilatório forneceu poucas informações prognósticas adicionais considerando todo o espectro de FEVE na IC. Tentando abordar a potencial relação dinâmica desta complexa interação biológica, investigamos associações não lineares para possíveis intervalos ou limites sob risco diferencial, em todo o espectro de ambas as exposições. Encontramos associação não linear entre $V F_{1} / C V F$ e o desfecho composto, em que indivíduos com IC com $\mathrm{VEF}_{1} / \mathrm{CVF}$ maior que $75 \%$ diminuíram a probabilidade de apresentar eventos cardiovasculares maiores em uma média de 2,2 anos de acompanhamento. Podemos supor que: 1) a DPOC, a principal causa de obstrução das vias aéreas que frequentemente coexiste com a $\mathrm{IC}$, poderia não ter sido detectada, aumentando a carga de risco; ${ }^{4}$ ou 2) dos efeitos primários da IC no sistema respiratório, as alterações do VEF podem ser mais sensíveis que a CVF em períodos mais curtos, influenciadas por alterações dinâmicas em brônquios de calibres pequenos e médios. ${ }^{23} \mathrm{~A}$ capacidade vital reduzida, como marca registrada da IC avançada, ${ }^{9}$ foi menos sensível para distinguir o risco de eventos incidentais ao longo deste tempo de acompanhamento.

Contribuições potenciais da função pulmonar para a incidência de eventos cardiovasculares também parecem diferir entre os fenótipos ICFEP e ICFER. Restringindo a análise a um subconjunto de indivíduos com LVEF $\leq 50 \%$, uma diminuição no $\mathrm{VEF}_{1} / C V F$, mas não na ppCVF, identificou maior risco para o desfecho composto, enquanto nenhuma conclusão pôde ser feita para aqueles com FEVE > 50\% com apenas dois eventos. Da mesma forma, no subconjunto de fraqueza inspiratória, a redução do $\mathrm{VEF}_{1} / \mathrm{CVF}$ distinguiu maior risco de eventos cardiovasculares maiores, o que não foi observado entre aqueles sem fraqueza ou em todo o espectro ppCVF. Poderíamos especular que a fisiopatologia do padrão obstrutivo impactou negativamente principalmente aqueles com FEVE reduzida e com fraqueza inspiratória.

\section{Limitações}

Várias limitações devem ser observadas. Como um estudo observacional, a causalidade não pôde ser abordada e podem existir fatores de confusão residuais e não medidos para as observações descritas. Apenas um subconjunto de pacientes foi incluído, com espirometria completa, necessária para este estudo e, dada a idade mais jovem daqueles excluídos, viés de seleção involuntário poderia estar presente. As medidas espirométricas foram realizadas sem broncodilatadores, de forma que a obstrução reversível permaneceu indetectável; além disso, os padrões restritivos foram baseados apenas na CVF, porque medidas mais precisas e diretas de volumes e capacidades não estavam disponíveis, o que poderia ter limitado a capacidade de detectar a verdadeira restrição de volume pulmonar, porém poderia aumentar a validade externa dos achados. Além disso, um mecanismo importante de limitação do exercício pode ser devido ao aprisionamento de ar, que não pode ser detectado pela espirometria.

Infelizmente, a VVM medida não foi viável para todos os participantes, o que pode ter influenciado métricas de reserva ventilatória, provavelmente subestimadas, embora apenas 5 participantes tiveram $<20 \%$ de reserva ventilatória. No entanto, trocamos resultados potencialmente não confiáveis, por valores uniformes e comparáveis em toda a coorte usando a medida de MVV estimada. Apesar de uma correlação razoável entre $\operatorname{FEV}_{1}$ e $\operatorname{MVV}\left(r^{2}=0,82\right),{ }^{36}$ reconhecemos que o VVM deve ser realizado antes do CPX em nível individual, sempre que possível.

Também reconhecemos que a baixa saturação de oxigênio durante o exercício pode representar um desacoplamento de ventilação-perfusão ou restrição ventilatória, o que não é exclusivamente, mas mais frequentemente associada ao comprometimento do sistema respiratório, particularmente DPOC avançada, doenças pulmonares intersticiais e hipertensão pulmonar, ${ }^{18}$ condições excluídas no início do estudo. No entanto, um oxímetro compatível com nosso sistema de CPX estava indisponível durante a aquisição de dados, e o transdutor digital existente produziu valores não confiáveis. Portanto, recorremos ao exame físico normal (sem sibilância ou cianose no pico de esforço) para supor que a hipóxia era improvável.

Por fim, o tempo de acompanhamento relativamente curto e, consequentemente, a taxa de eventos podem ter limitado a capacidade de detectar associações prognósticas com ppCVF, mas não com $V E F_{1} / C V F$, devido ao comportamento mais crônico do primeiro.

\section{Implicações para a prática clínica}

A espirometria e a manovacuometria são ferramentas de função pulmonar amplamente disponíveis, embora subutilizadas na IC. 4,26 A interpretação de defeitos ventilatórios pode ser desafiadora nesses indivíduos, particularmente naqueles com ICFEP, cuja variação fenotípica pode sobrepor sintomas cardíacos e pulmonares, e menos dados são disponíveis. ${ }^{9}$ No entanto, podem 
fornecer valiosas informações sobre o impacto da IC no sistema respiratório, diferenciando-se da doença pulmonar não diagnosticada (e subtratada), interpretando melhor a CPX, identificando potenciais alvos terapêuticos (reabilitação, treinamento ventilatório) e definindo fatores prognósticos, enfatizando que a espirometria é uma ferramenta disponível e viável, que deve ser realizada previamente ao CPX e para apoiar a estratificação de risco na IC. Alterações subclínicas e em estágios iniciais da função pulmonar podem predizer eventos cardiovasculares futuros. Somando-se a esse conhecimento, nosso estudo sugere que, também na IC mais crônica e estável, a presença e o tipo de disfunção pulmonar auxiliam na melhor interpretação das respostas ao exercício e na identificação de sujeitos sob maior risco.

\section{Conclusão}

Em uma coorte do mundo real com indivíduos com IC crônica, com ampla faixa de fração de ejeção, $V F_{1} / C V F$ e ppCVF foram diretamente associados à reserva ventilatória no exercício, levando em consideração a idade e o sexo. No entanto, apenas ppCVF reduzida foi adicionalmente associada a baixa fração de ejeção, fraqueza inspiratória e baixa capacidade de exercício. Em seguimento médio de 2,2 anos, apenas o $\mathrm{VEF}_{1} / \mathrm{CVF}$, mas não a ppCVF, distinguiu indivíduos com IC sob maior risco de eventos cardiovasculares maiores, sendo mais proeminentes entre aqueles com fração de ejeção reduzida e baixa pressão inspiratória. Portanto, $\mathrm{VEF}_{1} / \mathrm{CVF}$ e ppCVF adicionam informações distintas sobre os fenótipos de IC.

\section{Referências}

1. Neder JA, Rocha A, Berton DC, O'Donnell DE. Clinical and Physiologic Implications of Negative Cardiopulmonary Interactions in Coexisting Chronic Obstructive Pulmonary Disease-Heart Failure. Clin Chest Med. 2019;40(2):421-38. doi: 10.1016/j.ccm.2019.02.006.

2. Cundrle I Jr, Olson LJ, Johnson BD. Pulmonary Limitations in Heart Failure. Clin Chest Med. 2019;40(2):439-48. doi: 10.1016/j.ccm.2019.02.010.

3. Lalande S, Cross TJ, Keller-Ross ML, Morris NR, Johnson BD, Taylor BJ. Exercise Intolerance in Heart Failure: Central Role for the Pulmonary System. Exerc Sport Sci Rev. 2020;48(1):11-9. doi: 10.1249/JES.0000000000000208.

4. Canepa M, Straburzynska-Migaj E, Drozdz J, Fernandez-Vivancos C, Pinilla JMG, Nyolczas N, et al. Characteristics, Treatments and 1-year Prognosis of Hospitalized and Ambulatory Heart Failure Patients with Chronic Obstructive Pulmonary Disease in the European Society of Cardiology Heart Failure Long-Term Registry. Eur J Heart Fail. 2018;20(1):100-10. doi: 10.1002/ejhf.964.

5. Lawson CA, Mamas MA, Jones PW, Teece L, McCann G, Khunti K, et al. Association of Medication Intensity and Stages of Airflow Limitation With the Risk of Hospitalization or Death in Patients with Heart Failure and Chronic Obstructive Pulmonary Disease. JAMA Netw Open. 2018;1(8):e185489. doi: 10.1001/jamanetworkopen.2018.5489.

6. Damarla M, Celli BR, Mullerova HX, Pinto-Plata VM. Discrepancy in the Use of Confirmatory Tests in Patients Hospitalized with the Diagnosis of Chronic Obstructive Pulmonary Disease or Congestive Heart Failure. Respir Care. 2006;51(10):1120-4.

\section{Agradecimento}

Agradecemos a todos os pacientes que aceitaram participar do nosso estudo, doando-nos seu tempo e esperança de melhorar o entendimento da IC, em busca de uma assistência de melhor qualidade.

\section{Contribuição dos autores}

Concepção e desenho da pesquisa: Ramalho SHR, Lima ACGB, Cipriano GFB, Cipriano Junior G; Obtenção de dados: Ramalho SHR, Lima ACGB, Silva FMF, Souza FSJ, Cipriano GFB, Cipriano Junior G; Análise e interpretação dos dados: Ramalho SHR, Cahalin LP, Cipriano GFB, Cipriano Junior G; Análise estatística: Ramalho SHR; Obtenção de financiamento: Lima ACGB, Cipriano GFB, Cipriano Junior G; Redação do manuscrito: Ramalho SHR; Revisão crítica do manuscrito quanto ao conteúdo intelectual importante: Ramalho SHR, Lima ACGB, Silva FMF, Souza FSJ, Cahalin LP, Cipriano GFB, Cipriano Junior G.

\section{Potencial conflito de interesse}

Não há conflito com o presente artigo

\section{Fontes de financiamento}

O presente estudo foi parcialmente financiado pela Capes e CNPq.

\section{Vinculação acadêmica}

Este artigo é parte de tese de doutorado de Sergio Henrique Rodolpho Ramalho pela Universidade de Brasília.

7. Johnson BD, Beck KC, Olson LJ, O'Malley KA, Allison TG, Squires RW, et al. Ventilatory Constraints during Exercise in Patients with Chronic Heart Failure. Chest. 2000;117(2):321-32. doi: 10.1378/chest.117.2.321.

8. Tzani P, Piepoli MF, Longo F, Aiello M, Serra W, Maurizio AR, et al. Resting Lung Function in the Assessment of the Exercise Capacity in Patients with Chronic Heart Failure. Am J Med Sci. 2010;339(3):210-5. doi: 10.1097/ MAJ.0b013e3181c78540.

9. Magnussen H, Canepa M, Zambito PE, Brusasco V, Meinertz T, Rosenkranz S. What Can We Learn from Pulmonary Function Testing in Heart Failure? Eur J Heart Fail. 2017;19(10):1222-9. doi: 10.1002/ejhf.946.

10. Iversen KK, Kjaergaard J, Akkan D, Kober L, Torp-Pedersen C, Hassager C, et al. The Prognostic Importance of Lung Function in Patients Admitted with Heart Failure. Eur J Heart Fail. 2010;12(7):685-91. doi: 10.1093/eurjhf/ hfq050.

11. Andrea R, López-Giraldo A, Falces C, López T, Sanchis L, Gistau C, et al. Pulmonary Function Predicts Mortality and Hospitalizations in Outpatients with Heart Failure and Preserved Ejection Fraction. Respir Med. 2018;134:124-9. doi: 10.1016/j.rmed.2017.12.004.

12. Ramalho SHR, Shah AM. Lung Function and Cardiovascular Disease: A Link. Trends Cardiovasc Med. 2021;31(2):93-8. doi: 10.1016/j.tcm.2019.12.009.

13. Miller MR, Hankinson J, Brusasco V, Burgos F, Casaburi R, Coates A, et al. Standardisation of Spirometry. Eur Respir J. 2005;26(2):319-38. doi: 10.1183/09031936.05.00034805. 
14. Lang RM, Badano LP, Mor-Avi V, Afilalo J, Armstrong A, Ernande L, et al. Recommendations for Cardiac Chamber Quantification by Echocardiography in Adults: An Update from the American Society of Echocardiography and the European Association of Cardiovascular Imaging. J Am Soc Echocardiogr. 2015;28(1):1-39. doi: 10.1016/j.echo.2014.10.003.

15. Neder JA, Andreoni S, Castelo-Filho A, Nery LE. Reference Values for Lung Function Tests. I. Static Volumes. Braz J Med Biol Res. 1999;32(6):703-17. doi: 10.1590/s0100-879x1999000600006

16. Neder JA, Andreoni S, Lerario MC, Nery LE. Reference Values for Lung Function Tests. II. Maximal Respiratory Pressures and Voluntary Ventilation. Braz J Med Biol Res. 1999;32(6):719-27. doi: 10.1590/s0100879x1999000600007.

17. Caruso P, Albuquerque AL, Santana PV, Cardenas LZ, Ferreira JG, Prina E, et al. Diagnostic Methods to Assess Inspiratory and Expiratory Muscle Strength. J Bras Pneumol. 2015;41(2):110-23. doi: 10.1590/S180637132015000004474

18. Guazzi M, Adams V, Conraads V, Halle M, Mezzani A, Vanhees L, et al. EACPR/AHA Scientific Statement. Clinical Recommendations for Cardiopulmonary Exercise Testing Data Assessment in Specific Patient Populations. Circulation. 2012;126(18):2261-74. doi: 10.1161/ CIR.0b013e31826fb946.

19. Castello-Simões V, Minatel V, Karsten M, Simões RP, Perseguini NM, Milan JC, et al. Circulatory and Ventilatory Power: Characterization in Patients with Coronary Artery Disease. Arq Bras Cardiol. 2015;104(6):476-85. doi: 10.5935/abc.20150035

20. Barron AJ, Medlow KI, Giannoni A, Unsworth B, Coats AJ, Mayet J, et al. Reduced Confounding by Impaired Ventilatory Function with Oxygen Uptake Efficiency Slope and VE/VCO2 Slope Rather Than Peak Oxygen Consumption to Assess Exercise Physiology in Suspected Heart Failure. Congest Heart Fail. 2010;16(6):259-64. doi: 10.1111/j.17517133.2010.00183.x.

21. Barron A, Francis DP, Mayet J, Ewert R, Obst A, Mason M, et al. Oxygen Uptake Efficiency Slope and Breathing Reserve, Not Anaerobic Threshold, Discriminate between Patients with Cardiovascular Disease Over Chronic Obstructive Pulmonary Disease. JACC Heart Fail. 2016;4(4):252-61. doi: 10.1016/j.jchf.2015.11.003.

22. Ingle L, Shelton RJ, Cleland JG, Clark AL. Poor Relationship Between Exercise Capacity and Spirometric Measurements in Patients with More Symptomatic Heart Failure. J Card Fail. 2005;11(8):619-23. doi: 10.1016/j. cardfail.2005.06.430.

23. Hawkins NM, Virani S, Ceconi C. Heart Failure and Chronic Obstructive Pulmonary Disease: The Challenges Facing Physicians and Health Services. Eur Heart J. 2013;34(36):2795-803. doi: 10.1093/eurheartj/eht192.

24. Miyagi $M$, Kinugasa $Y$, Sota $T$, Yamada $K$, Ishisugi $T$, Hirai $M$, et al. Diaphragm Muscle Dysfunction in Patients with Heart Failure. J Card Fail. 2018;24(4):209-16. doi: 10.1016/j.cardfail.2017.12.004.
25. Nakagawa NK, Diz MA, Kawauchi TS, Andrade GN, Umeda IIK, Murakam FM, et al. Risk Factors for Inspiratory Muscle Weakness in Chronic Heart Failure. Respir Care. 2020;65(4):507-16. doi: 10.4187/respcare.06766.

26. Ribeiro JP, Chiappa GR, Neder JA, Frankenstein L. Respiratory Muscle Function and Exercise Intolerance in Heart Failure. Curr Heart Fail Rep. 2009;6(2):95-101. doi: 10.1007/s11897-009-0015-7.

27. Dall'Ago P, Chiappa GR, Guths H, Stein R, Ribeiro JP. Inspiratory Muscle Training in Patients with Heart Failure and Inspiratory Muscle Weakness: A Randomized Trial. J Am Coll Cardiol. 2006;47(4):757-63. doi: 10.1016/j. jacc.2005.09.052.

28. Yamada K, Kinugasa Y, Sota T, Miyagi M, Sugihara S, Kato M, et al. Inspiratory Muscle Weakness is Associated with Exercise Intolerance in Patients with Heart Failure with Preserved Ejection Fraction: A Preliminary Study. J Card Fail. 2016;22(1):38-47. doi: 10.1016/j.cardfail.2015.10.010.

29. Ramalho SHR, Cipriano G Jr, Vieira PJC, Nakano EY, Winkelmann ER, Callegaro CC, et al. Inspiratory Muscle Strength and Six-Minute Walking Distance in Heart Failure: Prognostic Utility in a 10 Years Follow up Cohort Study. PLoS One. 2019;14(8):e0220638. doi: 10.1371/journal. pone.0220638.

30. Tucker WJ, Haykowsky MJ, Seo Y, Stehling E, Forman DE. Impaired Exercise Tolerance in Heart Failure: Role of Skeletal Muscle Morphology and Function. Curr Heart Fail Rep. 2018;15(6):323-31. doi: 10.1007/s11897018-0408-6.

31. Kelley RC, Ferreira LF. Diaphragm Abnormalities in Heart Failure and Aging: Mechanisms and Integration of Cardiovascular and Respiratory Pathophysiology. Heart Fail Rev. 2017;22(2):191-207. doi: 10.1007/ s10741-016-9549-4.

32. Cuttica MJ, Colangelo LA, Dransfield MT, Bhatt SP, Rana JS, Jacobs DR Jr, et al. Lung Function in Young Adults and Risk of Cardiovascular Events Over 29 Years: The CARDIA Study. J Am Heart Assoc. 2018;7(24):e010672. doi: 10.1161/JAHA.118.010672.

33. Silvestre OM, Nadruz W Jr, Roca GQ, Claggett B, Solomon SD, Mirabelli MC, et al. Declining Lung Function and Cardiovascular Risk: The ARIC Study. J Am Coll Cardiol. 2018;72(10):1109-22. doi: 10.1016/j.jacc.2018.06.049.

34. Olson TP, Denzer DL, Sinnett WL, Wilson T, Johnson BD. Prognostic Value of Resting Pulmonary Function in Heart Failure. Clin Med Insights Circ Respir Pulm Med. 2013;7:35-43. doi: 10.4137/CCRPM.S12525.

35. Georgiopoulou VV, Deka A, Li S, Niazi AA, Farooq K, Kalogeropoulos AP, et al. Pulmonary Function Testing and Outcomes in Subjects with Heart Failure Listed for Heart Transplantation. Respir Care. 2015;60(5):731-9. doi: 10.4187/respcare.03709.

36. Dillard TA, Hnatiuk OW, McCumber TR. Maximum Voluntary Ventilation. Spirometric Determinants in Chronic Obstructive Pulmonary Disease Patients and Normal Subjects. Am Rev Respir Dis. 1993;147(4):870-5. doi: 10.1164/ajrccm/147.4.870

\section{* Material suplementar}

Para informação adicional, por favor, clique aqui. 